\title{
Towards Reverse Engineering of Industrial Site Plants
}

\author{
Abner M. C. Araújo ${ }^{1}$, Manuel M. Oliveira ${ }^{1}$ (Advisor) \\ ${ }^{1}$ Instituto de Informática - Universidade Federal do Rio Grande do Sul (UFRGS) \\ \{amcaraujo,oliveira\}@inf.ufrgs.br
}

\begin{abstract}
Computer-Aided Design (CAD) models of industrial sites are extremely important, as they provide documentation, and simplify inspection, planning, modification, as well as a variety of physical and logistics simulations. Despite these clear advantages, many industrial sites do not have CAD models, or have trouble keeping them up-to-date. This thesis presents some initial efforts towards developing an automatic framework for producing CAD models from point clouds obtained by scanning complex environments. It introduces fast and robust algorithms for detecting planes and cylinders in noisy unorganized point clouds that represent such installations. The detection and removal of such structures from the input point cloud should facilitate the detection of other components. We demonstrate the effectiveness of our techniques by comparing their performances against the state-of-the-art solutions and showing that ours achieved the best overall accuracy on real and synthetic datasets.
\end{abstract}

\section{Introduction}

CAD models are a key element in the design, construction, inspection, and maintenance of large, complex installations. Unfortunately, many existing installations do not have CAD models or have outdated ones. Moreover, for quality control, one needs to check the construction/assembly of modern environments against their designs. Thus, the ability to scan real environments and obtain corresponding CAD models is highly desirable.

In industrial sites, planes and cylinders are among the most common structures. They are used to model floors, walls, ceilings, pipes, tanks and ducts. However, detecting even such simple geometric structures in unorganized point clouds is a challenging task. Planes may appear in arbitrary directions and sizes, while cylinders may contain various radii, orientations, and lengths. Moreover, unorganized point clouds introduce additional challenges such as noise, non-uniform sampling density, incomplete models due to occlusions, and lack of semantic relationship among samples.

\subsection{Contributions}

This thesis introduces efficient solutions for automatic detection of planes and cylinders in unorganized point clouds. More specifically, we present an efficient $O(n \log n)$ deterministic technique for robust plane detection in unorganized point clouds. Our solution uses robust statistics to derive a novel planarity test that is insensitive to outliers. It uses robust measures of distance to plane and normal deviation to detect and remove outliers, as well as to automatically adjust its parameters to the local distribution of samples in the input dataset. This results in a robust-to-noise approach, which is also virtually independent of parameter tuning, and handles point clouds of large sizes and variable sampling distributions. To attain such goal, we introduce two additional contributions: an iterative grow-merge procedure capable of retrieving connected planar regions with a great 


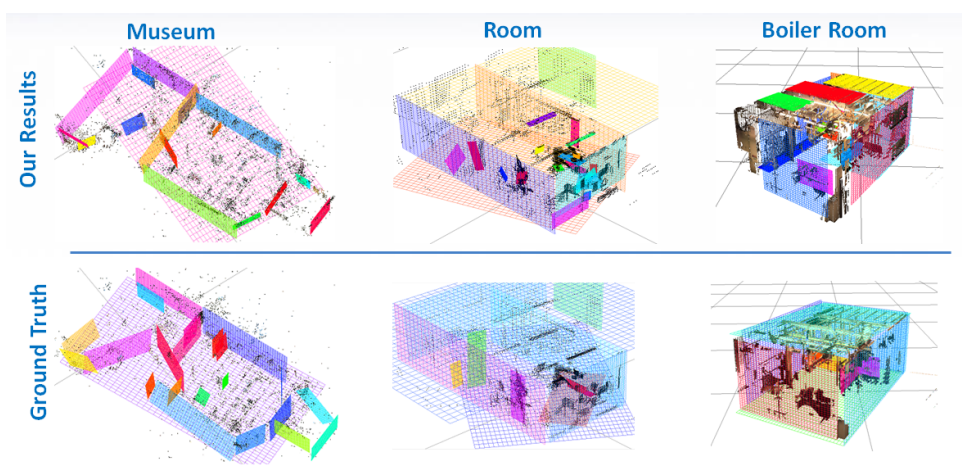

Figure 1. Examples of automatic plane detection in unorganized point clouds obtained by our technique and corresponding ground truths.

level of precision and detail; and a mechanism to automatically adjust the plane detection parameters in order to fit the local distribution of samples. A paper describing our robust plane detection technique and its associated contributions, entitled A Robust Statistics Approach for Plane Detection in Unorganized Point Clouds [Araujo and Oliveira 2020b], was published in the Pattern Recognition journal, one of the most prestigious in the field. Additional information about our plane-detection solution, including code and datatesets can be found in our plane detection project website. 1

Our second major contribution is a technique for fast cylinder-detection in unorganized point clouds that is robust to noise, uses parameters which require little to no fine-tuning, and can handle cylinders with arbitrary orientations. In order to develop such a technique, we also introduce a fast deterministic circle-recognition method capable of filtering noisy samples. These results are summarized in the paper Connectivity-based Cylinder Detection in Unorganized Point Clouds [Araujo and Oliveira 2020a] also published in the Pattern Recognition journal. Additional information can be found in our cylinder detection project website ${ }^{2}$

\section{Plane Detection}

Our plane-detection technique is based on robust statistics and has three main steps: split, grow, and merge. In the split phase, the input point cloud is spatially subdivided using an octree until the leaf nodes have less than $\epsilon$ samples ( $0.1 \%$ of the total samples). A robustto-noise planarity test (Section 2.1) is then applied to the samples of each leaf node. If they pass the test, they undergo a growth process and can further be merged with adjacent cells into larger planar patches. The growth parameters are automatically estimated from the data distribution itself. Finally, in the merge phase, patches are merged according to some boundary condition. The grow and merge phases are iterated until no patches can grow any further, when the algorithm outputs the set of detected planar regions (Figure 2).

\subsection{Planarity Test}

The standard procedure for testing co-planarity in a set of samples uses principal component analysis (PCA) to obtain an eigen-decomposition of the samples' covariance matrix [Limberger and Oliveira 2015, Li et al. 2017, Vo et al. 2015]. One way to check for

\footnotetext{
${ }^{1}$ http://www.inf.ufrgs.br/ oliveira/pubs_files/RE/RE.html

${ }^{2}$ http://www.inf.ufrgs.br/ oliveira/pubs_files/CD/CD.html
} 
(approximate) co-planarity is by comparing the ratio between the smallest and largest eigenvalue magnitudes. If such ratio is smaller than some threshold $\tau$, the samples are considered coplanar: $\left|\lambda_{1}\right| \leq\left|\lambda_{2}\right| \leq\left|\lambda_{3}\right|, \frac{\left|\lambda_{1}\right|}{\left|\lambda_{3}\right|}<\tau$. But, since PCA uses the covariance matrix, which is computed using the mean of each variable, it is sensitive to outliers. In statistics, breakdown point is the percentage of outliers an estimator can handle before giving incorrect results. The mean estimator is said to have a breakdown point of $0 \%$, since a single outlier can disturb it.

The thesis introduces a new co-planarity test that is robust to outliers and has linear cost in the number of samples. It is based on robust statistics alternatives to the mean and standard deviation: the median and the median absolute deviation (MAD). Both median and MAD estimators have breakdown points of $50 \%$, since it would require more than $50 \%$ of outlier observations to disturb them. MAD is computed as: $M A D=k \times$ median $\left(\mid x_{i}-\right.$ median $\left.(X) \mid\right)$, where $x_{i} \in X$ represents all individual samples in the set $X . k$ is a constant that makes MAD yield consistent results with standard deviation. For a normal distribution, $k=1.4826$ [Rousseeuw and Croux 1993]. Using these two estimators, we developed two tests to check whether a sample might belong to a plane: (i) plane-sample distance test, which checks if the sample distance to the estimated plane is inside a safe interval calculated as median $(D)+3 \times M A D(D)$, where $D=\left\{d_{1}, d_{2}, \ldots, d_{n}\right\}$ and $d_{i}$ is the orthogonal distance of sample $i$ the to estimated plane; and (ii) plane-sample normal deviation tests, which checks if the deviation of the sample normal with respect to the estimated plane normal is inside a safe interval calculated as median $(\Phi)+3 \times M A D(\Phi)$, where $\Phi=\left\{\phi_{1}, \phi_{2}, \ldots, \phi_{n}\right\}$ and $\phi_{i}$ is the angular difference between the normal of sample $i$ and the estimated plane normal. Additionally, we perform a third test to validate the plane itself: outlier-ratio test, which verifies if the percentage of outliers is below a certain threshold $(25 \%)$.

\section{Cylinder Detection}

In order to detect cylinders with arbitrary orientations, our technique projects the point cloud onto a set of uniformly-distributed directions on a unit hemisphere. Each direction defines a tangent plane onto which we orthographically project the samples whose normals are approximately perpendicular to the plane normal. For each group $g_{i}$ of projected samples that form a connected component in $3 \mathrm{D}$, we refine the orientation of the corresponding projection plane based on these samples's normals and re-project them onto the new plane. Then, a novel circle-recognition technique is applied to elements of $g_{i}$ to detect circular projections. Delimited cylindrical surfaces are then obtained by merging related connected components in 3D and fitting cylinders to the merged components (Figure 2).

\subsection{Circle Detection}

Unlike previous cylinder-detection techniques, ours is based on a circle detection technique that has linear cost in the number of projected samples. For each connected component in the projections, we check if it fits a circle. This is achieved by exploring the fact that extended normals from each sample of a circle should intersect at the circle's center. Since point clouds are susceptible to occlusion, our algorithm handles projections forming arcs of varying lengths. Our circle-detection technique is faster than RANSAC- and Hough-transform-based solutions, and does not require the specification of noise-level thresholds. Due to space restrictions, we refer the reader to [Araujo and Oliveira 2020a] or to the full thesis [Araujo 2019] for additional details. 


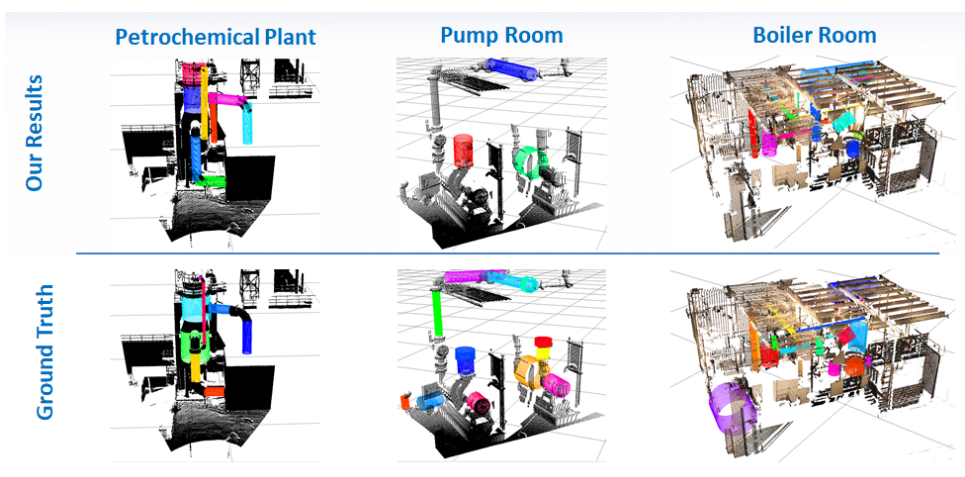

Figure 2. Examples of automatic cylinder detection in unorganized point clouds obtained by our technique and corresponding ground truths.

\section{Results}

We implemented our technique in C++ and used OpenGL to render the point clouds and the detected planes and cylinders. For our comparisons, we chose popular and recent works on plane and cylinder detection. Whenever available, we used the authors' own implementations. All techniques were compared using both synthetic and real datasets. We obtained the ground truths by manually labeling each dataset ourselves. The results for each combination of technique and dataset are displayed in Figures 3 and 4 . Our technique achieved competitive results for all evaluated datasets, and when considering the average precision, recall and F1 score, our methods surpassed the state-of-art for both plane and cylinder detection.

\section{Conclusions}

This thesis presented the first steps towards obtaining an automatic robust-to-noise framework for recovering CAD models from point clouds captured from complex environments, such as industrial site plants. We presented fast and robust techniques to detect planes and cylinders.

Our automatic $O(n \log n)$ plane-detection technique achieves better accuracy, measured in terms of average precision, recall, and F1 score, than the previous approaches, while still being one of the fastest. Our cylinder-detection technique achieved the best F1 score on all datasets. One should note that when comparing both techniques, the parameters used by the competing techniques were individually tuned for each dataset in order to produce their best results in each case. For our techniques, in turn, we used default parameter values for all datasets, showing our methods' robustness and independence to parameter tuning, and their ability to handle point clouds in general. Regarding noisy point clouds, we have investigated the use of popular noise filtering and outlier removal techniques as a pre-processing step. Unfortunately, they have undesirable side effects. Low-pass filtering tends to collapse nearby structures and smooth sharp edges. Outlier removal often discards good samples in low-sampled surfaces, compromising their reconstruction. This reinforces the importance of techniques that are robust to noise.

\section{Acknowledgments}

This work was sponsored by CNPq-Brazil (fellowships and grants 130895/2017-2, 312975/2018-0, 423673/2016-5) and by ONR Global (award N62909-18-1-2131). 

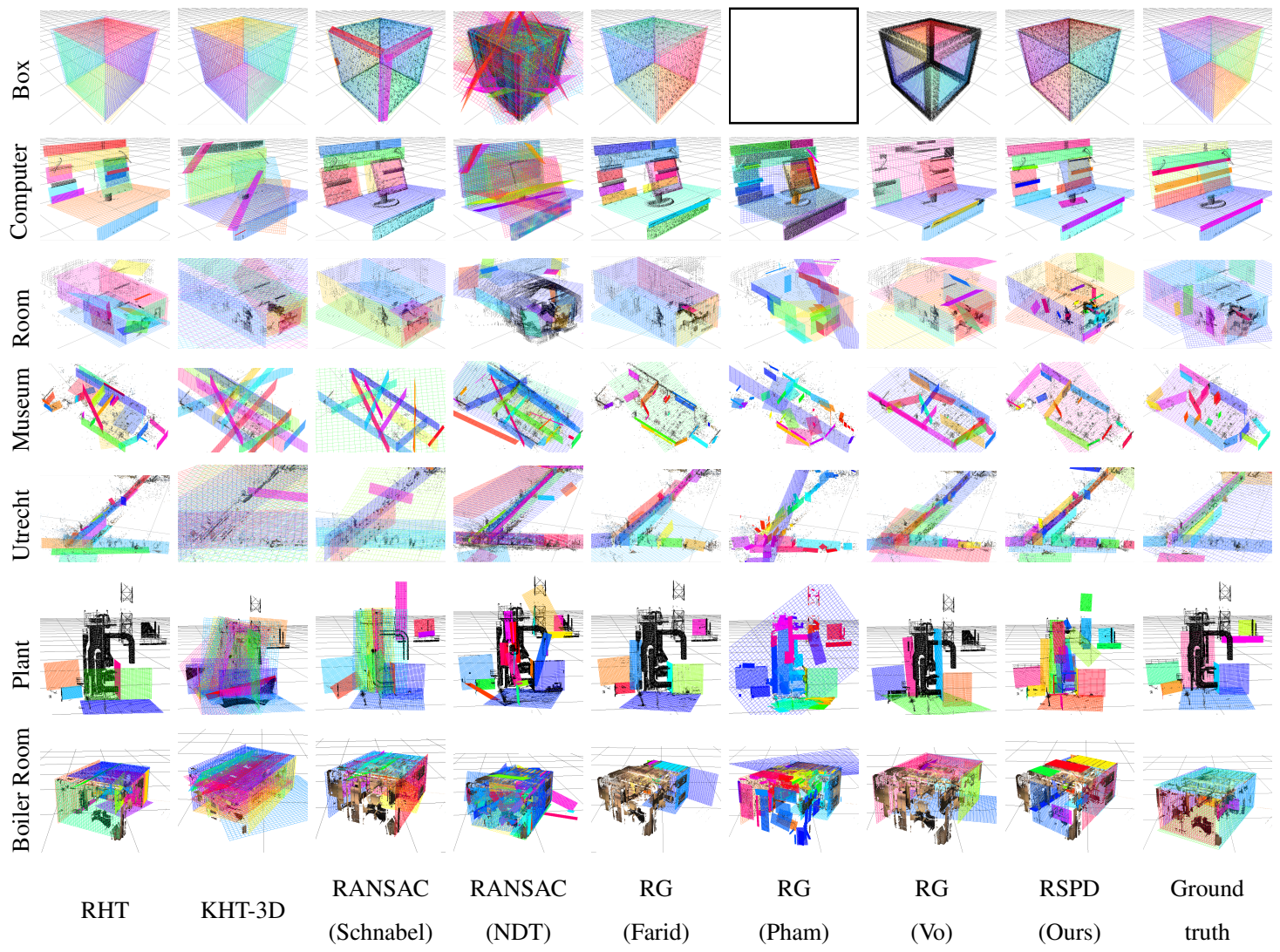

Figure 3. Planes detected by the compared techniques for all datasets. Ground truth is shown in the rightmost column. For each pair of technique and dataset, the detected planes have been highlighted using different colors. Black dots represent samples treated as outliers by each technique. We could not find a set of parameters to execute RG (Pham) on the Box dataset in reasonable time. RHT [Xu et al. 1990], KHT-3D [Limberger and Oliveira 2015], Schnabel [Schnabel et al. 2007], NDT [Li et al. 2017], Farid [Farid 2015], Pham [Pham et al. 2016], Vo [Vo et al. 2015].

\section{References}

Ahmed, M. F., Haas, C. T., and Haas, R. (2014). Automatic detection of cylindrical objects in built facilities. Journal of Computing in Civil Engineering, 28(3):04014009.

Araujo, A. M. C. (2019). Towards Reverse Engineering of Industrial Site Plants. Master's thesis, UFRGS, Porto Alegre, Brazil.

Araujo, A. M. C. and Oliveira, M. M. (2020a). Connectivity-based cylinder detection in unorganized point clouds. Pattern Recognition, 100:1-12. Article 107161.

Araujo, A. M. C. and Oliveira, M. M. (2020b). A robust statistics approach for plane detection in unorganized point clouds. Pattern Recognition, 100:1-12. Article 107115.

Farid, R. (2015). Region-growing planar segmentation for robot action planning. In Australasian Joint Conference on Artificial Intelligence, pages 179-191. Springer.

Li, L., Yang, F., Zhu, H., Li, D., Li, Y., and Tang, L. (2017). An improved RANSAC for $3 \mathrm{~d}$ point cloud plane segmentation based on normal distribution transformation cells. Remote Sensing, 9(5):433. 


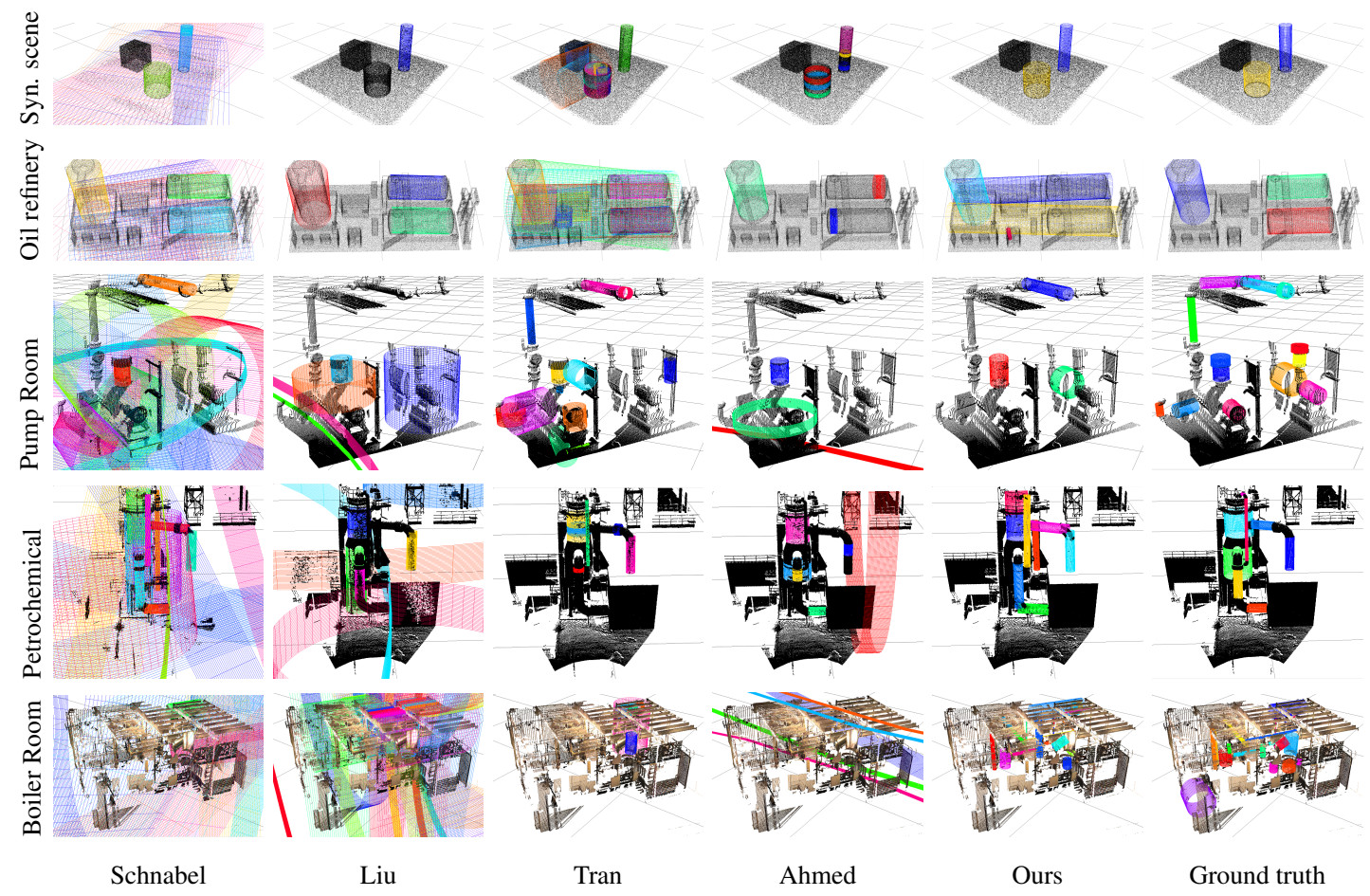

Figure 4. Cylinders detected by the compared techniques for all datasets. Ground truth is shown in the rightmost column. For each pair of technique and dataset, the detected cylinders have been highlighted using different colors. Black dots represent samples treated as outliers by each technique. Schnabel [Schnabel et al. 2007], Tran [Tran et al. 2015], Ahmed [Ahmed et al. 2014].

Limberger, F. A. and Oliveira, M. M. (2015). Real-time detection of planar regions in unorganized point clouds. Pattern Recognition, 48(6):2043-2053.

Pham, T. T., Eich, M., Reid, I., and Wyeth, G. (2016). Geometrically consistent plane extraction for dense indoor $3 \mathrm{~d}$ maps segmentation. In Intelligent Robots and Systems (IROS), 2016 IEEE/RSJ International Conference on, pages 4199-4204. IEEE.

Rousseeuw, P. J. and Croux, C. (1993). Alternatives to the median absolute deviation. Journal of the American Statistical association, 88(424):1273-1283.

Schnabel, R., Wahl, R., and Klein, R. (2007). Efficient RANSAC for point-cloud shape detection. In Computer graphics forum, volume 26, pages 214-226.

Tran, T.-T., Cao, V.-T., and Laurendeau, D. (2015). Extraction of cylinders and estimation of their parameters from point clouds. Computers \& Graphics, 46:345-357.

Vo, A.-V., Truong-Hong, L., Laefer, D. F., and Bertolotto, M. (2015). Octree-based region growing for point cloud segmentation. ISPRS Journal of Photogrammetry and Remote Sensing, 104:88-100.

Xu, L., Oja, E., and Kultanen, P. (1990). A new curve detection method: randomized Hough transform (rht). Pattern recognition letters, 11(5):331-338. 